\title{
Positron Studies of Subsurface Zone in Titanium Created in Sliding Wear
}

\author{
Jerzy Dryzek • Mirosław Wróbel
}

Received: 17 April 2014/ Accepted: 1 July 2014/Published online: 15 July 2014

(C) The Author(s) 2014. This article is published with open access at Springerlink.com

\begin{abstract}
Positron annihilation studies of the subsurface zone constituted during dry sliding in pure titanium are reported. A new type of defect depth profile in this zone, which is not an exponential decay usually reported for other metals and alloys, is observed. Two layers in the subsurface zone are present in this metal. In the layer adjacent to the worn surface, the characteristic plateau of the mean positron lifetime that extends to a depth of $60-70 \mu \mathrm{m}$ was observed. Small vacancy clusters and dislocations were recognized in this layer. At deeper zone, the mean positron lifetime decreased exponentially in the manner typical for the other metals. In this zone, vacancy clusters increase in size and decreases in concentration with increasing depth. There is a slight increase in measured microhardness in the subsurface zone in relation to the interior.
\end{abstract}

Keywords Titanium $\cdot$ Subsurface zone $\cdot$ Positron annihilation

\footnotetext{
J. Dryzek $(\bowtie)$

Institute of Physics, Opole University, ul. Oleska 48, 45-052 Opole, Poland

e-mail: jerzy.dryzek@ifj.edu.pl

J. Dryzek

Institute of Nuclear Physics PAN, ul. Radzikowskiego 152,

31-342 Kraków, Poland

M. Wróbel

AGH University of Science and Technology, 30 Av.

Mickiewicza, 30-059 Kraków, Poland
}

\section{Introduction}

Due to their high friction coefficient, titanium and its alloys exhibit poor tribological properties. This results from their chemical activity and strong adhesion to the countermaterial surface in frictional contact [1,2]. Additionally, similar to other $h c p$ metals, the main deformation mode of titanium is a basal slip on the (0001) plane and no mechanism of plastic deformation (i.e. slip and/or twinning) produces plastic strain only in the direction parallel to the $c$-axis of the unit cell. However, the authors reported that the slip plane of $a+c$ is active, as it is in polycrystalline titanium deformed at room temperature; the slip plane of $a+c$ dislocations $\{10 \overline{1} 1\}$ is reported in Ref. [3]. This causes the low number of slip systems which impedes the implementation of deformation. In our research, we investigate another factor which can affect the tribological properties of titanium, i.e. the subsurface zone (SZ) which is generated during sliding.

The SZ is the hardening region adjacent to the worn surface formed as the result of interaction of the countermaterial being in a friction contact [4]. Generally, the SZ exhibits increased hardness which decreases with the distance from the worn surface. This zone contains some defects generated mainly by the plastic deformation. They extend into the interior of the sample to the depth of dozens of micrometers, depending both on the deformation and the deformation rate. Different experimental methods are applied to the SZ studies, including scanning and transmission electron microscopy, X-ray diffraction, microhardness and the positron annihilation techniques which is particularly suitable for quantitative studies of the structure defects. These techniques allowed us to establish that measured characteristics, like a mean positron lifetime or 
S-parameter, which are sensitive to the open volume detect concentration, decrease exponential with increasing depth. It practically means that also the defect concentration decreases exponential of with increasing depth, a good example and discussion one can find in Ref. [5]. This dependence seems to be general feature for the SZ created during sliding. The large extent of the defect profile is surprising as well. Its total depth can extend to hundreds of micrometers, and it depends on the sliding condition (i.e. applied load, sliding velocity and duration) and hardly depends on the material properties (e.g. hardness). Up to now, we performed the SZ studies for following pure metals: $\mathrm{Bi}$ [5], $\mathrm{Cu}$ [6], $\mathrm{Al}$ [7], $\mathrm{Mg}$ [8], $\mathrm{Fe}$ [9] and some alloys (i.e. stainless steel [10], aluminium and magnesium alloys [11]).

Despite poor tribological properties, titanium seems to be interesting for general studies of the formation and constitution of the SZ. In the presented studies, we use the positron lifetime spectroscopy, Doppler broadening of annihilation line and microhardness measurement as complementary methods. Similar to other studies, the surface of the titanium sample is exposed to dry sliding contact and the SZ profiled using the sequenced etching technique.

\section{Experimental Details}

\subsection{Sample Preparation}

Discs of $99.99 \%$ pure polycrystalline titanium $10 \mathrm{~mm}$ in diameter and $3 \mathrm{~mm}$ thick were the subject of our studies. They were polished mechanically with $\mathrm{SiC}$ papers and $1-\mu \mathrm{m}$-particle-size diamond paste and then annealed in flowing $\mathrm{N}_{2}$ gas in a furnace at $700{ }^{\circ} \mathrm{C}$ for $1 \mathrm{~h}$. After annealing, the samples were etched in a solution of $10 \mathrm{ml}$ $\mathrm{HNO}_{3}, 2 \mathrm{ml} \mathrm{HF}$ and $10 \mathrm{ml}$ distilled water to reduce their thickness by ca. $20 \mu \mathrm{m}$ and to clean their surface. The sample preparation procedure described above allowed us to remove any defects induced in its manufacture. Such samples were treated as virgin ones at least in respect to the residual defects. For such virgin samples, the positron lifetime spectrum exhibited only one lifetime component of $147.9 \pm 0.5 \mathrm{ps}$, which corresponds well with the experimental values reported by other authors as the bulk value, i.e. $150 \mathrm{ps}$ [12], $149.0 \pm 1.0 \mathrm{ps}$ [13] or $152 \mathrm{ps}$ [14]. The view under microscope revealed that the samples are polycrystalline with large equiaxial grains. Their average size is an order of $100 \mu \mathrm{m}$.

Each virgin sample was located in a tribotester and its flat base surface was slid against a rotating disc made from the martensitic steel (steel HS 18-0-1 hardness, about 670 HV0.1, $50 \mathrm{~mm}$ in diameter) with a certain load applied. The treatment was performed in the air; no oxidation was observed. The velocity of the disc relative to the surface of the sample was $5 \mathrm{~cm} / \mathrm{s}$. The sliding duration was $1 \mathrm{~min}$ for each sample. The value of the friction coefficient obtained was 0.31 , and the specific wear rate, defined as a worn volume per unit load, was $(4.23 \pm 0.02) \times 10^{-13} \mathrm{~m}^{3} \mathrm{~N}^{-1} \mathrm{~m}^{-1}$.

\subsection{Positron Lifetime Measurements}

For the positron lifetime measurement, a fast-fast positron lifetime spectrometer with $\mathrm{BaF}_{2}$ scintillator was used. The time resolution (FWHM) of the spectrometer was 250 ps. The positron source, i.e. ${ }^{22} \mathrm{Na}$ enveloped in the $7-\mu \mathrm{m}$ kapton foil was located between the worn surfaces of the two identical pieces of samples, and this sandwich was positioned in front of the scintillator detectors of the positron lifetime spectrometer. The positron lifetime spectrum was measured to obtain more than $2 \times 10^{6}$ counts in the spectrum. The average implantation depth, which is the reciprocal value of the linear absorption coefficient, for positrons emitted from ${ }^{22} \mathrm{Na}$ in $\mathrm{Ti}$ is ca. $48 \mu \mathrm{m}$. This value indicates the depth from the measured surface which is scanned by positrons in each measurement. About $76 \%$ of emitted positrons annihilate in the layer of such a thickness as simulated by the LYS-1 computer code [15].

As a supplemental measurement, for one sample, we performed also the measurement of the depth profile of the annihilation line shape parameter (so called S-parameter). This parameter is defined as the integral of central part of the annihilation line normalized to total integral of the line centred at $511 \mathrm{keV}$. It is well proved experimentally that this parameter is extremely sensitive to the presence of open volume defects, like the vacancy or its clusters and jogs at dislocation line, where due to their positive charge, positrons are localized. The measurements were performed using the HpGe detector with the energy resolution at the $511 \mathrm{keV}$ of $1.4 \mathrm{keV}$.

For detection of the defect depth distribution, i.e. detection of the SZ profile, the samples were sequenceetched, sequentially removing layers about $10 \mu \mathrm{m}$ thick from the worn surface, and the positron lifetime spectrum or line shape parameter were measured. The samples were etched in a solution similar to that described in Sect. 2.1. The etching procedure was repeated subsequently until the bulk value was reached.

\subsection{Microhardness Measurements}

Any changes of the microhardness in the subsurface region were investigated by producing cross section of samples using a diamond saw and then gently polishing them with Struers abrasive diamond paste. The depth profile of the microhardness was measured below the worn surface on the cross-sectional sample using a TUKON ${ }^{\mathrm{TM}} 2500$ (Instron) device. The measurements were performed 
according the standard ISO 4545 using the Knoop intender with the normal load of $0.1 \mathrm{~N}$. Each indentation was made in such a way that the longest diameter of the intender was parallel to the worn surface. All results were recalculated to the standard Vickers hardness unit.

\section{Results and Discussion}

\subsection{Positron Lifetime Measurements}

Two components in the positron lifetime spectra were observed for all samples exposed to dry sliding. Let us describe in detail results for sample whose surface was exposed to dry sliding with a load of $100 \mathrm{~N}$. Depending on the depth, the value of the first lifetime component varies in the range from 200 to $140 \mathrm{ps}$ and the second one from 200 to $280 \mathrm{ps}$. It is convenient to begin the analysis of the obtained results using a commonly accepted robust parameter which is the mean positron lifetime defined as follows:

$\bar{\tau}=\tau_{1} I_{1}+\tau_{2} I_{2}$,

where $\tau_{1,2}$ are the positron lifetime components resolved from the positron lifetime spectra, and $I_{1,2}$ are their intensities $\left(I_{1}+I_{2}=1\right)$. In Fig. 1 (closed circles) we depict its value as a function of the depth from the worn surface. As observed for other metals [4-8], the mean positron lifetime decreases with increasing depth. This is a characteristic feature of the SZ. However, in this case, in the layer directly adjacent to worn surface, the mean positron lifetime remains almost constant, i.e. about 190 ps. The thickness of this layer, which we call the first layer (FL), is about $60-70 \mu \mathrm{m}$. After that a second layer is observed where the value of the mean positron lifetime decreases with increasing depth. We call it the deeper layer (DL). It seems that the SZ consists of two layers, i.e. FL and DL and its total thickness induced by dry sliding for this sample is about $250 \mu \mathrm{m}$. The exponential decrease which was found for other studied metals and alloys is not observed [2-6]. In order to confirm this unexpected result, we performed the measurements of the S-parameter for the same samples. The value of the S-parameter conveys information complementary to that provided by the mean positron lifetime; however, they are measured with different spectrometers. The obtained dependency is depicted in Fig. 1 (closed circles). Both measurements revealed almost identical type of dependency, including the plateau adjacent to the worn surface layer. In Fig. 2, we depict in detail, the mean positron lifetime together with the lifetime components obtained from the deconvolution of the measured spectra at different depths. In the FL, the value of the second lifetime component, $\tau_{2}$, is about $250 \mathrm{ps}$ with the

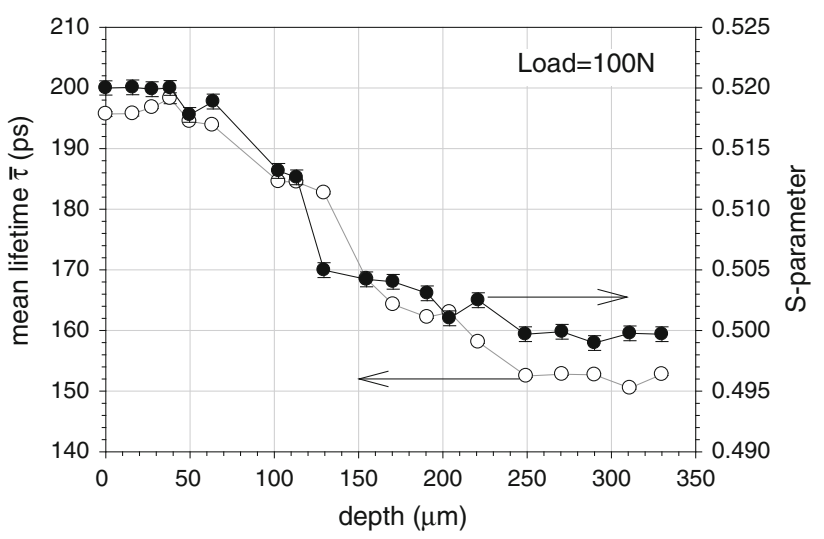

Fig. 1 The depth profile of the mean positron lifetime and value of the S-parameter measured for a pure titanium sample exposed to dry sliding against steel with an applied load of $100 \mathrm{~N}$
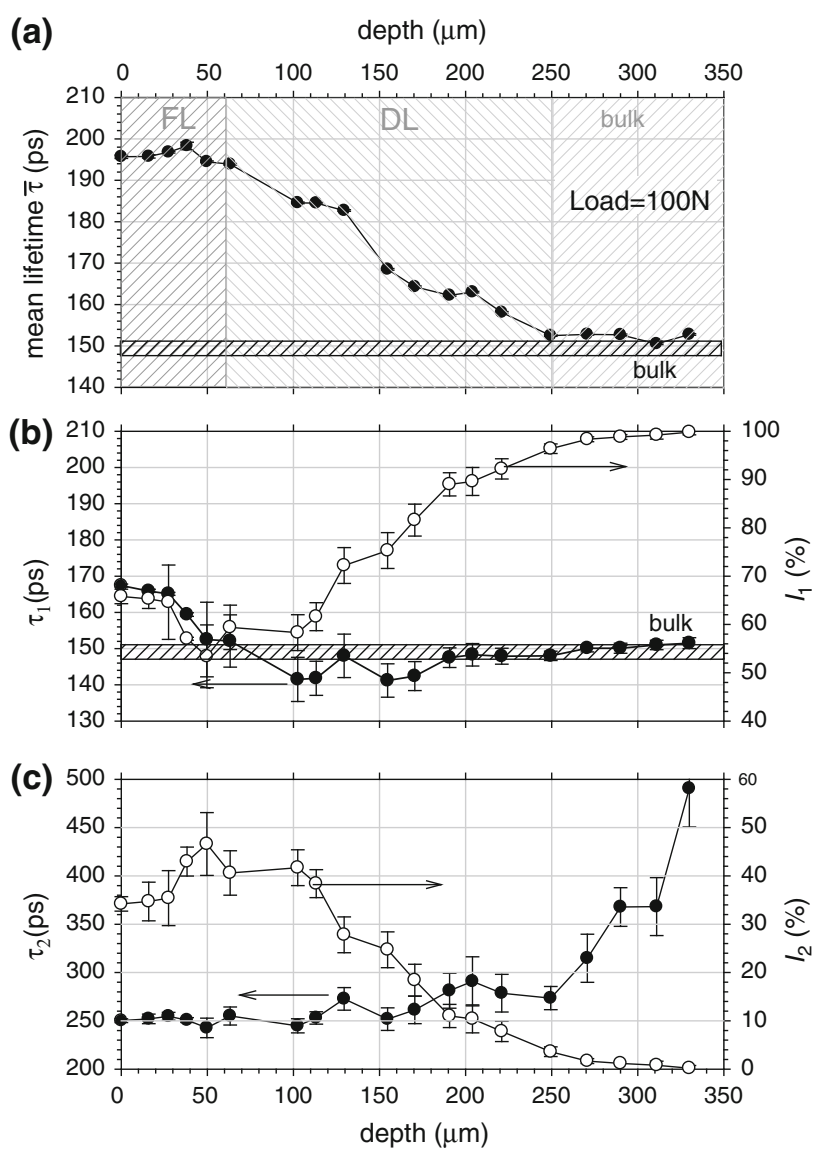

Fig. 2 The depth profile of the positron lifetime components, $\tau_{1}(\mathbf{b})$, $\tau_{2}(\mathbf{c})$, and their intensities $I_{1}(\mathbf{b}), I_{2}(\mathbf{c})$ and the mean positron lifetime (a) obtained from the deconvolution of the positron lifetime spectra measured for the pure titanium sample exposed to dry sliding against steel with the applied load of $100 \mathrm{~N}$. The FL, DL and bulk region are tagged by hatched lines

high intensity of about $30-45 \%$, Fig. 2c. The value of the positron lifetime in a single vacancy in a perfect host in titanium is reported to be 222 ps [12], slightly lower than 
our value. Thus, one can conclude that in this layer, the vacancy clusters containing two or three vacancies are present. In the FL, the value of the first lifetime component, $\tau_{1}$, decreases from ca. 170 to 150 ps which is close to the bulk value, Fig. 2 b. Simultaneously, its intensity also decreases from ca. 65 to $55 \%$. At the depth above $100 \mu \mathrm{m}$, the value of $\tau_{1}$ is slightly lower than bulk value, and with the increase of the depth, its value increases again to the bulk value whereas its intensity increases significantly from 48 to $100 \%$ at the depth of $300 \mu \mathrm{m}$. The $\tau_{2}$ value up to the depth of $250 \mu \mathrm{m}$ increases slightly from 250 to 280 ps. It indicates that in the DL region at the depth between 60 and $250 \mu \mathrm{m}$, the vacancy clusters size increases, but their concentration decreases with the depth from the worn surface. In the bulk region, above $250 \mu \mathrm{m}$, the $\tau_{2}$ value increases to the value more than $400 \mathrm{ps}$, meanwhile its intensity decreases to zero at the depth of $300 \mu \mathrm{m}$. However, this large increase of the $\tau_{2}$ value can be treated as an artefact caused by the deconvolution procedure of

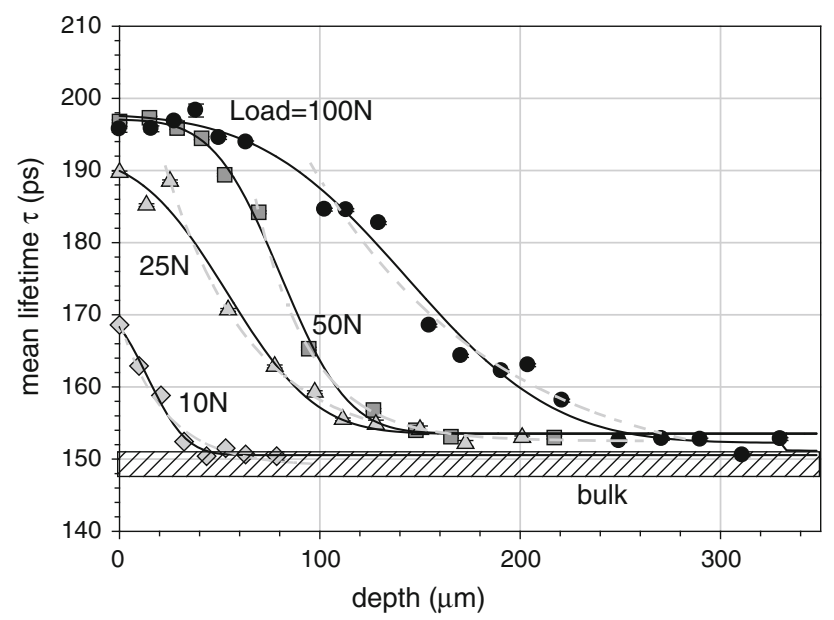

Fig. 3 The depth profile of the mean positron lifetime obtained for the pure titanium samples exposed to dry sliding against steel with different applied loads. The black solid lines represent the best fit of the Eq. 2 to the experimental points. The adjustable parameters from this equation are gathered in Table 1 . The dashed, grey lines represent the best fit of the exponential decreasing function, i.e. $\bar{\tau}=a+$ $b \exp \left(-z / d_{0}\right)$ in the region of the DL positron lifetime spectra. It can happen when the intensity of the component is very low.

The FL is characterized by the decreases of the value of the first lifetime component and the constant value of second component. We argue that this layer is highly deformed characterized by high concentration of the small vacancy clusters and jogs at dislocation lines where positrons are localized. The obtained depth dependencies for the DL can be understood in the frame of two-state trapping model [16] commonly used in the positron spectroscopy. In this model, positrons can annihilate in the bulk as free particles or localized at a defect. This causes that the first lifetime component is lower than the bulk value. This is a characteristic feature of the two-state trapping model. Thus, the DL must contain vacancy clusters but also the virgin regions where positrons can annihilate as free particles. A similar dependency was observed in bismuth samples exposed to dry sliding [5]. However, in this case, no FL was observed and the value of the mean positron lifetime, and consequently the defect concentration decreased exponentially starting from the worn surface.

The existence of the FL in the SZ in titanium is confirmed by measurement for other titanium samples after sliding with lower loads. The obtained depth profiles of the mean positron lifetime are depicted in Fig. 3. It can be noticed that the thickness of the FL decreases with the decrease of the applied load, and for the load of about $10 \mathrm{~N}$, it disappears. For this load, the obtained dependency is the exponential decay function detected for other metals. Certainly, we can confidently support the idea that SZ in titanium contains two separate layers; however, we found that the obtained dependences in Fig. 3 can be described using a simply sigmoidal formula:

$\bar{\tau}=a+\frac{b}{2}\left[1+\operatorname{erf}\left(\frac{c-z}{\sqrt{2} d}\right)\right]$

where $z$ is depth from the worn surface, and $a, b, c$ and $d$ are the adjusted parameters which values can be obtained in the fitting procedure. The $c$ parameter is the depth at which the sigmoidal curve reaches the transition centre and $b$ is the transition height. The solid lines in Fig. 3 present results of the best fit of Eq. 2 to the experimental points, and it can be noted that the experimental data are well described. In Table 1, we gather
Table 1 The values of the adjusted parameters in Eq. (2) used for description of the obtained depth dependency of the mean positron lifetime depicted in Fig. 3

In the last row the total depth of the SZ in the studied samples is presented

\begin{tabular}{lllll}
\hline & $100 \mathrm{~N}$ & $50 \mathrm{~N}$ & $25 \mathrm{~N}$ & $10 \mathrm{~N}$ \\
\hline$a(\mathrm{ps})$ & $152.2(1.2)$ & $153.6(1.0)$ & $153.5(1.1)$ & $154.5(1.0)$ \\
$b(\mathrm{ps})$ & $48.1(0.9)$ & $43.5(0.6)$ & $38.8(2.3)$ & $21.6(2.5)$ \\
$c(\mu \mathrm{m})$ & $143.8(4.8)$ & $80.7(1.7)$ & $54.1(7.1)$ & $14.0(5.6)$ \\
$d(\mu \mathrm{m})$ & $58.3(7.0)$ & $26.4(3.0)$ & $34.8(8.0)$ & $15.2(4.7)$ \\
Total depth $(\mu \mathrm{m})$ & 250 & 150 & 130 & 30 \\
Approximate thickness of the FZ & 80 & 60 & 50 & 50 \\
$\quad$ from microhardness profile & & & & \\
\hline
\end{tabular}




\section{(a)}
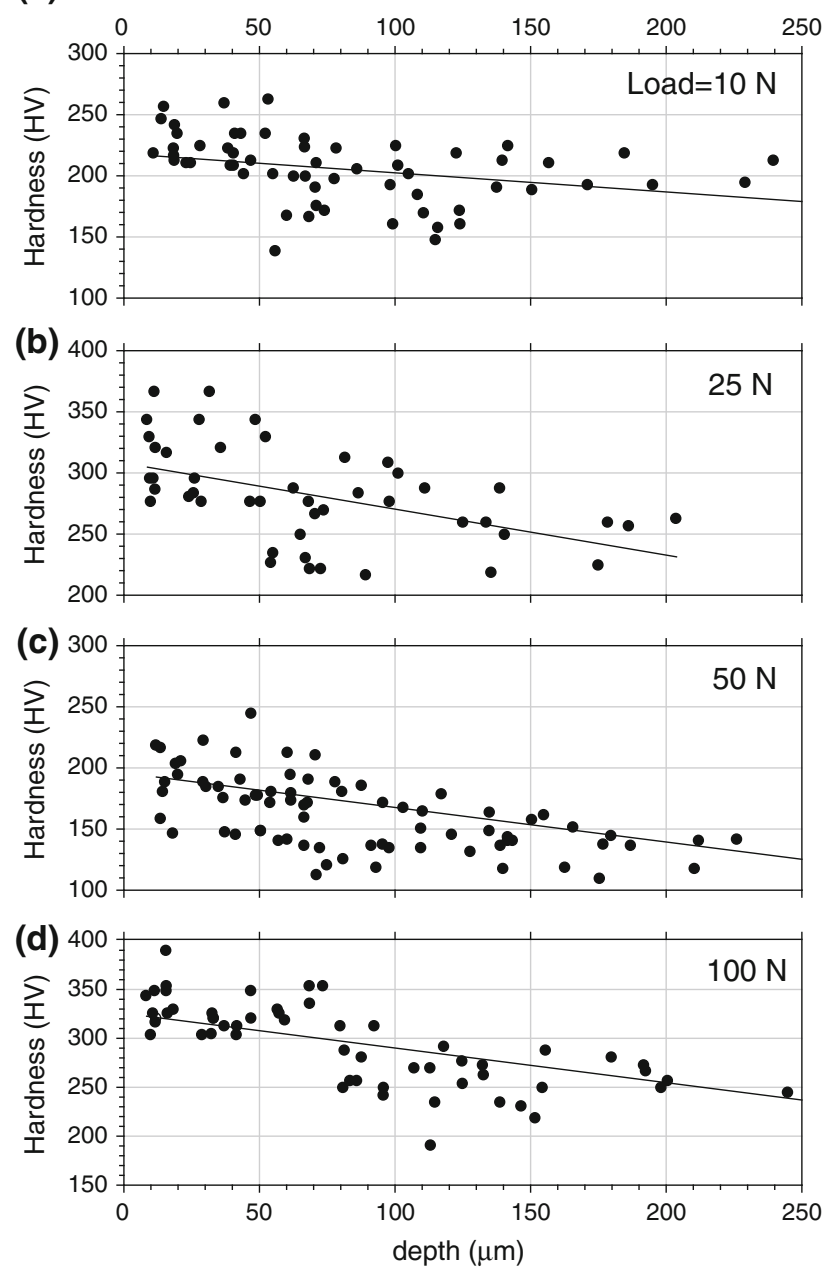

Fig. 4 The depth profile of the microhardness obtained for the pure titanium samples exposed to dry sliding against steel with different applied loads. The straight lines represent the least square regression lines fitted to the experimental points

the values of parameters together with the values of the total depth of the SZ (defined as the depth where the bulk value of the mean positron lifetime is reached) determined from Fig. 3. It means the deeper regions were not affected by the sliding processes at the surface. From Table 1, it is evident that the total depth value decreases with the decrease of the applied load. This tendency was noticed for other metals as well, see for example Ref. [9]. The dependency of the $b, c$ and $d$ parameter on the applied load is also clearly visible in Table 1.

\subsection{Microhardness Measurement}

In the literature, it has been reported that the SZ can be characterized by the microhardness profile measurements [4]. This method seems to be appropriate because hardness is a measure of the resistance to local plastic deformation, and the SZ is workhardening zone. However, as microhardness is a local property dependent on the crystallographic orientation of the measured area and grains, determination of a credible microhardness profile is labourintensive and not precise method, as was shown in our previous paper [10]. This was also confirmed for titanium in the present research. The microhardness depth profiles for the samples after sliding with different loads are depicted in Fig. 4. The measured points exhibit a large scatter; nevertheless, the linear regression lines added to Fig. 4 indicate the weak tendency of the microhardness to decrease with increasing depth. The slope of the linear regression lines depends slightly on the sliding load value; for the load of $24 \mathrm{~N}$, it is about -0.156 , and for the load of $100 \mathrm{~N}$, this value decreases to -0.354 . Moreover, the increase in the microhardness in the layer directly adjacent to the worn surface is visible. It is more clearly discernable for the highest sliding load of $100 \mathrm{~N}$. In this case, the total depth of this zone can be estimated to be ca. $80 \mu \mathrm{m}$. For the other sliding loads investigated, i.e. from 10 to $50 \mathrm{~N}$, the total depth of this zone can be estimated to be close to $50 \mu \mathrm{m}$. This result in some sense correlates with the FL region detected by positron measurements. Thus, we can conclude that the presence of the FL in the SZ was confirmed in the microhardness profiles, but resolution of this method was not sufficient to determine precisely of the FL thickness.

It should be noticed that the hardening due to sliding shown in the microhardness profile is very weak. For instance for the load of $100 \mathrm{~N}$, Fig. 4d, the microhardness at the depth of $15 \mu \mathrm{m}$ from the worn surface is ca. $330 \mathrm{HV}$, but in the bulk is about $250 \mathrm{HV}$. Thus, the increase is about $32 \%$. For the stainless steel $1.4301(\mathrm{EN})$, the increase of the microhardness was $72 \%$, see Fig. 5 in Ref. [10]. Thus, the workhardening of the SZ for titanium is rather weak; then, we suppose that this can explain also the poor tribological properties of this metal.

\subsection{Discussion and Remarks}

We explain the constitution of the SZ as the result of dislocations flowing from the worn surface into the material interior. These dislocations are generated at the plastically deformed asperities or from the plastic deformation in the regions adjacent to the worn surface. Dislocations with jogs during their motion are the source of point defects, i.e. interstitial atoms and vacancies, and these can form clusters which are detected by the positron annihilation experiments [17]. The concentration of such microstructure defects gradually decreases with the depth from the worn surface. Usually, the exponential function describes quite well the defect concentration decreasing with the depth from the worn surface. However, in titanium, another type 
of mean positron lifetime depth profile was observed. One can expect that dislocations generated during sliding accumulate, for reasons unknown, close to the worn surface creating the FL with a thickness increasing with the sliding load up to ca. $80 \mu \mathrm{m}$ for the largest sliding load applied, i.e. $100 \mathrm{~N}$. The large thickness of this layer is a puzzling fact.

Nevertheless, in the DL, the defect concentration decreases with the depth similarly to the other metals and alloys studied in the literature, see for example Ref. [5]. One can speculate that the depth dependency in the DL can be also described using an exponentially decreasing function, i.e. $\bar{\tau}=a+b \exp \left(-z / d_{0}\right)$. Indeed, the dashed, grey lines in Fig. 3 represent the best fit of such a function to the experimental points, and it also shows good agreement, but this applies only to DL and not to the whole SZ.

We can try to link the presence of the FL with the poor tribological properties of titanium. However, the friction coefficient against the steel is about 0.31 , and the wear for this metal is about $(4.23 \pm 0.3) \times 10^{-13} \mathrm{~m}^{3} / \mathrm{Nm}$. This value is higher than that for stainless steel 1.4301 (EN), $(2.01 \pm 0.12) \times 10^{-13} \mathrm{~m}^{3} / \mathrm{Nm}[10]$, and lower than that for pure iron, $(5.6 \pm 0.11) \times 10^{-13} \mathrm{~m}^{3} / \mathrm{Nm}$ [9], measured in identical conditions. However, another hcp metal, i.e. magnesium, measured by us exhibits the higher wear$(8.0 \pm 0.8) \times 10^{-13} \mathrm{~m}^{3} / \mathrm{Nm}-[8]$. It should be noted that for all these metals, no FL's was observed, contrary to the case of titanium investigated in the present research. However, at this stage, it is difficult to link the presence of the FL with poor tribological properties because there is no evidence sufficiently strong enough to confirm such a conclusion.

The existence of the FL can be also explained as the result of the saturation of positron trapping in defects in this region. If the trapping rate coefficient at vacancy clusters is very high, it is possible that even for low cluster concentration, all positrons are localized, and the saturation of positron mean lifetime is observed. No experimental data on positron trapping in titanium are available; however, studies performed by Hood and Schultz [18] indicate that saturation trapping occurs after a thickness reduction of $15 \%$. For example for stainless steel, it is observed at much lower value, i.e. at $3 \%$ reduction in thickness and no FL is observed [10]. Serra and De Diego [19] observed only a single lifetime component of $194 \pm 2$ ps in a titanium sample exposed to the cold rolling with the thickness reduction about $28 \%$. They attributed this lifetime to zone dislocations present at twin boundaries, and they supported it by computer simulations. This result encouraged us to investigate highly deformed titanium, i.e. cold rolled up to ca. $90 \%$ reduction of thickness. In the measured positron lifetime spectrum, only a single lifetime component $183.2 \pm 0.4 \mathrm{ps}$ was revealed. These values are different from those obtained for dry sliding, where at the worn surface, two components were detected, indicating the presence of vacancy clusters. Thus, it is difficult to support the saturation effect being responsible for the existence of the SZ.

There is another interesting fact concerning the FL. One can speculate that this layer can disappear after long time of sliding. However, we found that a sample exposed to the sliding during $12 \mathrm{~min}$ also exhibits in the defect depth profile with the FL. Thus, this zone is a characteristic feature of the SZ in titanium.

It is interesting to note that the small three-dimensional clusters of two to three vacancies were observed by Nancheva et al. [20] in pure titanium and titanium alloy BT14 samples exposed to an explosive wave. The maximum initial wave pressure in this experiment was about $20 \mathrm{GPa}$ which occurs within approximately $10^{-9} \mathrm{~s}$. The authors were able to resolve two lifetime components in their spectra (e.g. $\tau_{1}=185 \pm 5 \mathrm{ps}$ and $\tau_{2}=290 \pm 21 \mathrm{ps}$, the latter had the intensity about $19 \pm 7 \%$ ). These values correspond well to our data. The passage of a shock wave through a material involves a generation of large numbers of dislocations in the shock front. These dislocations can generate further dislocations but by the more usual multiplication process. Large numbers of dislocations must lead to the creation of different point defects including vacancies which finally coalescence into clusters. This can support the idea of the SZ constitution presented above. One can suppose that high pressure can only occur at asperities at the worn surface during the short time of order microseconds or less. These conditions correspond to the condition at a shock front but on a much smaller scale. Nevertheless, this can lead to similar vacancy-clusterrelated results to those seen in the positron lifetime spectra. They do not occur during static compression as it was shown above.

\section{Conclusions}

In the SZ of titanium samples exposed to dry sliding, the positron lifetime measurements revealed the presence of vacancy clusters whose size slightly increased with the depth from the worn surface. However, in the layer directly adjacent to the worn surface, dislocations were also detected. It is interesting that, in this layer, the value of the positron mean lifetime remains almost constant. This encouraged us to postulate that the SZ consists of two layers, the first one where the positron mean lifetime is almost constant and the second one where it decreases with depth from the worn surface. The thickness of the first layer is less than $100 \mu \mathrm{m}$ and strictly depends on the load applied during dry sliding. Nevertheless, the obtained depth dependence of mean positron lifetime can be described by 
a sigmoidal binding curve (Eq. 2). An increase in the microhardness in the layer adjacent to the worn surface was also observed; however, it was rather weak in comparison with other metals.

Acknowledgments The authors express their gratitude to Ewa Dryzek for fruitful discussion.

Open Access This article is distributed under the terms of the Creative Commons Attribution License which permits any use, distribution, and reproduction in any medium, provided the original author(s) and the source are credited.

\section{References}

1. Heinrichs, J., Olsson, M., Jacobson, S.: Transfer of titanium in sliding contacts-new discoveries and insights revealed by in situ studies in the SEM. World Tribology Congress, Tourino 2013. ISBN 978-88-908185

2. Garbacz, H., Gradzka-Dahlke, M., Kurzydłowski, K.J.: The tribological properties of nano-titanium obtained by hydrostatic extrusion. Wear 263, 572-578 (2007)

3. Numakura, H., Mjnontshi, Y., Koiwa, M.: $\langle\overline{1} \overline{1} 23\rangle\{10 \overline{1} 1\}$ slip in titanium polycrystals at room temperature. Scripta Metall 20, 1581-1586 (1986)

4. Zum Gahr, K.-H.: Microstructure and wear of materials. Elsevier, Amsterdam (1987)

5. Dryzek, J.: Positron studies of subsurface zone created in sliding wear in bismuth. Tribol. Lett. 40, 175-180 (2010)

6. Dryzek, J., Dryzek, E., Stegemann, T., Cleff, B.: Positron annihilation studies of subsurface zones in copper. Tribol. Lett. 3, 269-275 (1997)

7. Dryzek, J., Dryzek, E.: Subsurface zone in aluminium studied by positron lifetime spectroscopy. Tribol. Lett. 17, 147-153 (2004)
8. Dryzek, J., Dryzek, E., Suzuki, T., Yu, R.: Subsurface zone in pure magnesium studied by positron lifetime spectroscopy. Tribol. Lett. 20, 91-97 (2005)

9. Dryzek, J.: Positron studies of subsurface zone in pure iron induced by sliding. Tribol. Lett. 42, 9-15 (2011)

10. Dryzek, J., Horodek, P., Wróbel, M.: Use of positron annihilation measurements to detect the defect beneath worn surface of stainless steel $1.4301(\mathrm{EN})$ under dry sliding condition. Wear 294-295, 264-269 (2012)

11. Dryzek, J., Dryzek, E.: Subsurface zone studies in the AK 12 alloy and metal matrix composite AK 12 with $\mathrm{Al} 2 \mathrm{O} 3$ using positron annihilation spectroscopy. Wear 261, 549-555 (2006)

12. Campillo Robles, J.M., Ogando, E., Plazaola, F.J.: Positron lifetime calculation for the elements of the periodic table. J. Phys. 19, 176222-(20 pp) (2007)

13. Djourelov, N., Misheva, M.: Source correction in positron annihilation lifetime spectroscopy. J. Phys.: Condens. Matter 8, 2081-2088 (1996)

14. Yli-Kappila, J., Moser, P., Kunzi, H., Hautojärvi, P.: Positron lifetime measurements on electron irradiation damage in amorphous $\mathrm{Pd}_{80} \mathrm{Si}_{20}$ and $\mathrm{Cu}_{50} \mathrm{Ti}_{50}$. Appl. Phys. A 27, 31-33 (1982)

15. http://www.ifj.edu.pl/ $\sim$ mdryzek/index.html

16. Krause-Rehberg, R., Leipner, H.S.: Positron Annihilation in Semiconductors. Springer, Berlin (1999)

17. Hull, D.: Introduction to dislocations. Pergamon Press, London (1975)

18. Hood, G.M., Schultz, R.J.: A study of deformation of $\mathrm{Zr}$ and $\mathrm{Ti}$ by positron annihilation spectroscopy. J. Nuclear Mater. 120, 94-98 (1984)

19. Serra, A., De Diego, N.: Characterization of defects in deformed titanium. Phys. Stat. Sol. (a) 110, 409-414 (1988)

20. Nancheva, N.M., Saarinen, K., Popov, G.S.: Positron annihilation in shock loaded titanium and titanium alloys BT14. Phys. Stat. Sol. (a) 95, 531-536 (1986) 